\title{
Colloidal Modified Fluid Gelatin Plasma
} Substitute

National Cancer Institute

\section{Source}

National Cancer Institute. Colloidal Modified Fluid Gelatin Plasma Substitute. NCI

Thesaurus. Code C153429.

A colloidal, isotonic, physiologically balanced, plasma volume substitute containing succinylated gelatine and electrolytes, with potential nephroprotective properties. Upon intravenous administration, colloidal modified fluid gelatin plasma substitute expands the blood volume, restores intravascular volume and increases blood flow, thereby preventing hypovolemia, hypotension and shock and may decrease the accumulation of nephrotoxic agents in the kidneys. 